\title{
Digital Image Watermarking Technique using High Frequency Band based on Discrete Wavelet Transform and Singular Value Decomposition
}

\author{
Ramandeep Kaur \\ Research Scholar \\ SBSSTC, Ferozepur
}

\author{
Sonika Jindal \\ Assistant Professor \\ SBSSTC, Ferozepur
}

\begin{abstract}
Fast growth in digital media, digital data (such as image, video, audio or text) are stored, transmitted and distributed through internet without any loss or damage of content. Digital image watermarking is use to protect data from manipulations. Watermarking not only allow user to access, view and interpret the image but also protects the ownership of the content, as protection is an important crucial area.

In this paper, a new digital composite image watermarking algorithm based on DWT-SVD that is robust against various attacks is proposed. We obtained four different frequency images using DWT and IDWT transform. Watermark is embedded in high frequency sub-band by modifying the singular value of image. Scaling factor is also used to improve the results. The experimental results demonstrate that new proposed algorithm withstand under different attacks.
\end{abstract}

\section{Keywords}

Discrete Wavelet Transform; Inverse Discrete Wavelet Transform; Singular Value Decomposition; Digital Image Watermarking; Poisson Signal to Noise Ratio; Mean Square Error; Correlation Coefficient.

\section{INTRODUCTION}

In the recent years, development in sharing of multi-media data including images, audios and videos in computer communications, makes data protection crucial area. Digital watermarking is a technique to remove these issues.

Digital watermarking is a process in which watermark is embedded in original data as a signal for protection which can later extract. In [1] various technique of digital watermarking is proposed. Embedding should be imperceptible and there should be no degradation to original as well as embedded data.

The watermarking techniques can be classified into following categories: spatial domain, transform domain and spread spectrum. In spatial domain techniques the watermark embedding is achieved by directly modifying the pixel values of the host image. The most commonly used method is Least Significant Bit (LSB) method. In transform domain techniques the host image is first converted into frequency domain by transformation method such as DCT, DFT, DWT, etc then, transform domain coefficients are modified by the watermark. Another technique is spread spectrum in which the basic idea is to spread the watermark energy over visually important frequency bands, so that the energy in any one band is small and undetectable, making the watermark imperceptible.
Recently, singular value decomposition (SVD) is explored as a new transform technique for watermarking. Researchers developed various SVD based watermarking techniques continuously, and these proposed techniques is more effective when compared to the previous techniques

In this paper we propose the Hybrid scheme for embedding of the watermark which is used in the most common image processing applications like Image compression, image watermarking, etc. In [2] proposed a hybrid image watermarking in their paper shows that embedding is done by modifying the singular value of original image. Human Visual system is proposed using hybrid image watermarking in [3]. A new reference watermarking using DWT-SVD is proposed in [4]. Further, hybrid image watermarking is proposed in $[5,6]$ where embedding is done in high frequency sub band of image. The paper is organized as follows: Section 2 describes the different terminologies used in the paper followed by the proposed technique in section 3. Section 4 demonstrates the experimental results followed by different attacks applied on the watermarked images. The results are discussed in detail in section 5 . The paper is concluded in section 6 followed by the references.

\section{TERMINOLOGY}

\subsection{Discrete wavelet transform}

Discrete Wavelet Transform (DWT) is a multi-resolution mathematical tool for hierarchically decomposing an image and can efficiently implement using different digital filters. Digital filters divide the input image into four nonoverlapping multi-resolution sub-image, a lower resolution approximation image (LL), horizontal (HL), vertical (LH) and diagonal $(\mathrm{HH})$ detail components. This process can be repeated to obtain multiple scale wavelet decomposition. The information in low frequency district is an image (LL) is close to the original image. The frequency districts of $\mathrm{HL}, \mathrm{LH}$ and $\mathrm{HH}$ respectively represents the upright detail, level detail and the diagonal detail of the original image. According HVS, human eyes are sensitive to the change of smooth district of image, but not sensitive to the small change of edge, profile and streak. Embedding the watermark in the higher level sub bands increases the robustness of the watermark. 


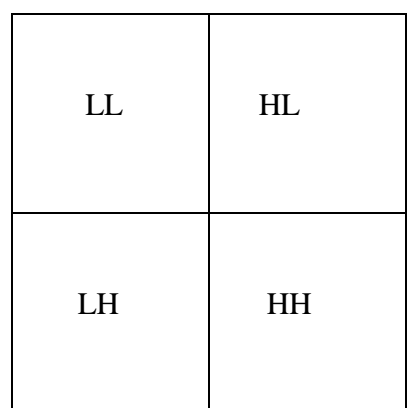

Figure 1. Wavelet Decomposition

\subsection{Singular Value Decomposition}

SVD is an effective numerical analysis tool used to analyze matrix of image. Using SVD transformation, a matrix can be decomposed into three matrices that are of the same size as the original matrix image. From the view point of linear algebra, an image is an array of nonnegative scalar entries that can be regarded as a matrix. Without loss of generality, if A is a square image, then SVD of A is defined as A=UASAVAT, where $\mathrm{U}$ and $\mathrm{V}$ are orthogonal matrices and $\mathrm{S}$ is a diagonal matrix.

SVD is an optimal matrix decomposition technique in a least square sense that it packs the maximum signal energy into as few coefficients as possible.

\section{PROPOSED TECHNIQUE}

We proposed a new digital image watermarking scheme. In this method we embed watermark in high frequency band of image by modifying the singular value and develop a new hybrid digital image watermarking scheme that shows the robustness and imperceptibility.

Assume the dimensions of the original image $A$ is $\mathrm{M} \times \mathrm{N}$ and dimensions of the visual watermark $W$ is $\mathrm{N} \times \mathrm{N}$.

\subsection{Watermark Embedding Process}

1. Using discrete wavelet transform (DWT), original image $A$ is decomposed into four sub-bands $L L_{a}, L H_{a}, H L_{a} a n d H H_{a}$ as shown in Figure1.

2. Apply IDWT to high frequency sub-band $\mathrm{HH}_{a}$.

3. Apply SVD to high frequency sub-band :

$$
H H_{a}=U_{a}^{h} S_{a}^{h} V_{a}^{h}
$$

4. Using discrete wavelet transform decompose watermark image $W$, into four sub-bands:

$$
L L_{w}, L H_{w}, H L_{w} \text { and HH }
$$

5. Apply IDWT to $H H_{w}$ sub-band of watermark image.

6. Apply SVD to high frequency sub-band:

$$
H H_{w}=U_{w}^{h} S_{w}^{h} V_{w}^{h}
$$

7. Modify the singular value :

$$
S_{a}^{* h}=\tanh \left(\left(\text { alpha } * S_{w}^{h}\right) / S_{a}^{h}\right)
$$

Where alpha is a scaling factor.

8. Apply SVD on obtained singular value:

$$
S_{a}^{* h}=U_{a}^{h} S_{a}^{h} V_{a}^{h}
$$

9. Using DWT to $S_{a}^{* h}$, obtain $H H_{a}^{*}$

$$
H H_{a}^{*}=\operatorname{DWT}\left(S_{a}^{* h}\right)
$$

10. Apply IDWT to obtain watermarked cover image $A^{*}$ using $L L_{a}, L H_{a}, H L_{a}$ and $H H_{a}^{*}$.

\subsection{Watermarking Extraction Process}

1. Decompose the watermarked image $A^{*}$ into four subbands using DWT:

$$
L L_{a}, H_{a}, H L_{a} \text { and } H H_{a}^{*} \text {. }
$$

2. Apply IDWT to high frequency sub-band:

$$
H H_{a}^{*}
$$

3. Apply SVD to high frequency sub-band $H H_{a}^{*}$

$$
H H_{a}^{*}=U_{w a}^{h} S_{w a}^{h} V_{w a}^{h}
$$

4. Apply SVD to High Frequency sub band of Watermark Image:

$$
H H_{w}=U_{w}^{h} S_{w}^{h} V_{w}^{h}
$$

5. Extract the singular value of high frequency sub-band watermark image:

$$
S_{w}^{* h}=a \tanh \left(\left(S_{w a}^{h} * S_{a}^{h}\right) / \text { alpha }\right)
$$

6. Using $S_{w}^{* h}$ recover the high frequency sub band of watermark image:

7. Recover the watermark image by using high frequency band with other existing sub band.

\section{EXPERIMENTAL RESULTS}

The experimental simulation is carried out using matlabR2010b. For standard test $256 \times 256,8$ bit grayscale images were used for studying the effects of imperceptibility and robustness of algorithm with scaling factor 0.2. The imperceptibility and robustness are the properties that are evaluated for the proposed scheme. Imperceptibility means that the superficial quality of the original image should not be distorted even after presence of watermark image. On the other hand, the robustness is the measure of the intentional and unintentional attacks. The Poisson signal-to-noise ratio (PSNR) and mean square error (MSE) is used to measure the quality between original image and watermarked image. 


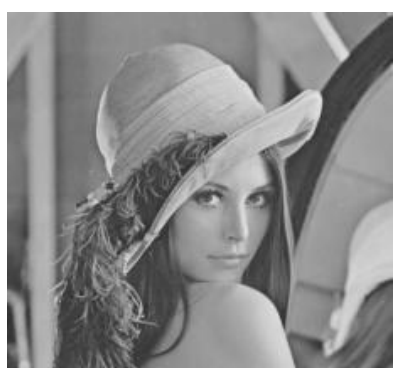

a) Lena

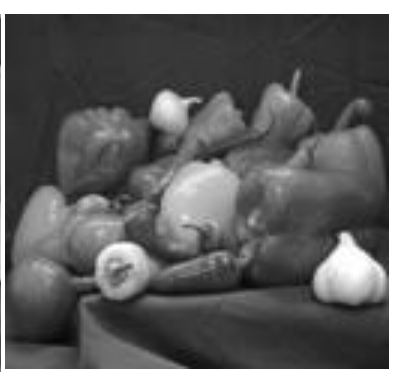

b) Peppers

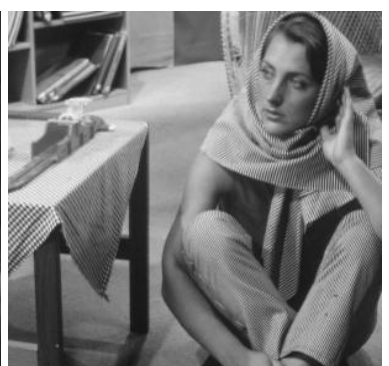

c) Barbara

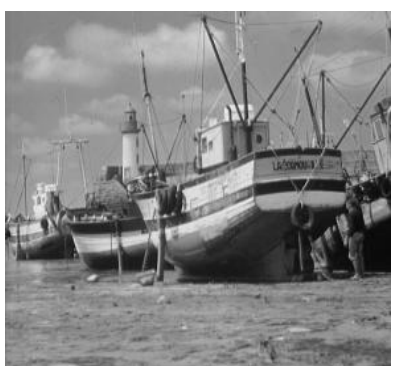

d) Boat

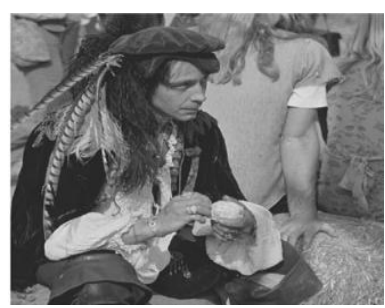

e) Pirate

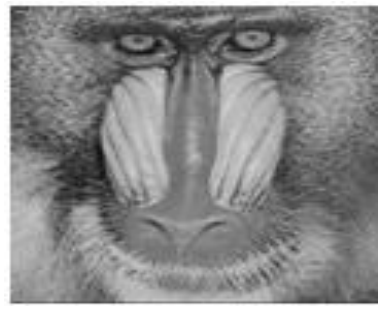

f) Mandrill

Figure 2 a) to e) Original Images 2f) watermark image

The MSE can be described as the mean of the square of the differences in the pixel values between the corresponding pixels of the two images. For the robust capability, mean absolute error (MSE) measures the mean of the square of the original watermark and the extracted watermark from the attacked image. The lower the value of the MSE lower will be the error.

$$
M S E=\frac{1}{M N} \sum_{i=0}^{M-1} \sum_{i=0}^{N-1}\left[\{x(i, j)-y(i, j)\}^{2}\right]
$$

The PSNR is used to evaluate the difference between original image and the watermarked image. PSNR value generally lies between $20 \mathrm{db}$ to $50 \mathrm{db}$.

$$
P S N R=10 \log 10\left(\frac{255 * 255}{M S E}\right)
$$

Correlation Coefficient (CC) is used to detect the similarity between original watermark and extracted watermark.

The standard test images are Lena, Peppers, Barbara, Boat and Pirate respectively shown as in Figure 2 a) to e). Figure $2 \mathrm{f})$ mandrill shows the watermark image. Table I shows the results for the PSNR, MSE and CC for various images after embedding the watermark.

Table1. Shows the PSNR, MSE and NC after embedding the watermark

\begin{tabular}{|c|c|c|c|}
\hline Images & PSNR(db) & MSE & CC \\
\hline Lena & 40.3962 & 5.9354 & 0.99824 \\
\hline Peppers & 43.7349 & 2.7516 & 0.99936 \\
\hline Barbara & 36.0351 & 8.9435 & 0.99707 \\
\hline Boat & 38.6157 & 16.2018 & 0.99785 \\
\hline Pirate & 39.1924 & 7.8313 & 0.99833 \\
\hline
\end{tabular}

\section{CONCLUSION}

The proposed watermarking algorithm is a new technique of watermarking which is also called semi blind watermarking technique as only original watermark and the algorithm is required for the detection of content. The PSNR lies between 20 to $50 \mathrm{db}$. CC is close to 1.0000 . There is always a trade-off between higher robustness and degree of perceptibility in the existing watermarking algorithms. The proposed algorithm achieves both robustness and imperceptibility. Further embedding can be done in any sub band of the image.

\section{REFERENCES}

[1] Langelaar, G.C., Setyawan, I., Lagendijk, R.I., 2000. "Watermarking digital image and video data", IEEE Signal Process. Magazine (September), 20-46.

[2] Ganic E, Eskicioglu AM.,"Robust embedding of visual watermarks using DWT-SVD” Journal of Electronic Imaging 2005;14(4).

[3] Bhatnagar Gaurav, Raman Balasubramanian."A new robust reference logo watermarking scheme",Multimedia Tools and Applications 2011;52(2):621e40.

[4] Li Q, Yuan C, Zong YZ.”Adaptive DWT-SVD domain image watermarking using human visual model", In: Proceedings of IEEE International Conference on Advanced Communication Technology (ICACT-2007), vol. 3; 2007. pp.1947e51.

[5] K. Bhagyashri and Joshi M.Y.,"Robust Image Watermarking based on Singular Value Decomposition and Discrete Wavelet Transform", Nanded C2010 IEEE.

[6] Ramandeep Kaur and Sonika Jindal, "Semi Blind Image Watermarking Using High Frequency Band Based on DWT-SVD", Sixth International Conference on Emerging Trends in Engineering and Technology (ICETET 2013) Nagpur, December 2013, pp 19 - 24. 